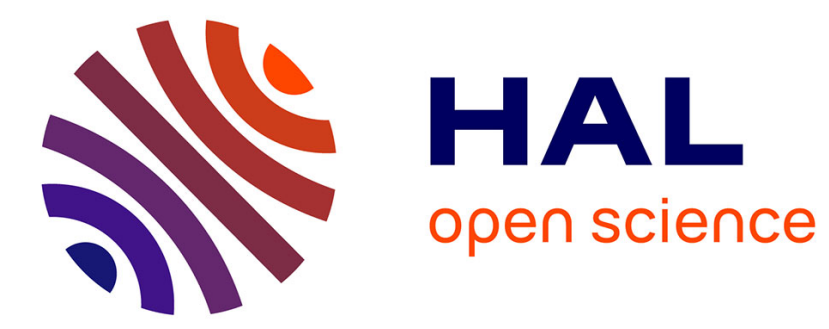

\title{
Récits ordinaires et textes stratégiques
}

Valérie-Inès de La Ville, Eléonore Mounoud

\section{To cite this version:}

Valérie-Inès de La Ville, Eléonore Mounoud. Récits ordinaires et textes stratégiques. Revue Française de Gestion, 2005, 159, 10.3166/rfg.159.343-358 . hal-01231078

\section{HAL Id: hal-01231078 \\ https://hal.science/hal-01231078}

Submitted on 10 Dec 2019

HAL is a multi-disciplinary open access archive for the deposit and dissemination of scientific research documents, whether they are published or not. The documents may come from teaching and research institutions in France or abroad, or from public or private research centers.
L'archive ouverte pluridisciplinaire HAL, est destinée au dépôt et à la diffusion de documents scientifiques de niveau recherche, publiés ou non, émanant des établissements d'enseignement et de recherche français ou étrangers, des laboratoires publics ou privés. 


\title{
Récits ordinaires et textes stratégiques
}

\author{
Valérie-Inés de La Ville delaville@iae.univ-poitiers.fr
}

et

Eléonore Mounoud eleonore.mounoud@ecp.fr

\section{Résumé}

Prendre en compte la nature textuelle des productions stratégiques de l'organisation conduit à souligner le rôle fondamental de la lecture dans la formation de la stratégie. La dynamique des textes stratégiques s'inscrit dans un double mouvement: d'une part, ils se constituent récursivement à partir des récits ordinaires liés aux activités quotidiennes et, d'autre part, ils interagissent avec les textes produits par les parties prenantes engagées par le devenir de l'organisation.

\section{Introduction : Le récit en management stratégique, au-delà d'un effet de mode...}

L'engouement que suscite la question du récit en management stratégique est marqué par un intérêt univoque pour la production des récits comme facteur d'efficacité dans la mise en œuvre des idées stratégiques de leurs auteurs. Ainsi, cette thématique a généré un ensemble de travaux centrés principalement sur les discours stratégiques (tant ceux des dirigeants que celui des théories) sans que les apports de l'approche narrative de l'organisation à la réflexion stratégique ne soient pris en compte. Les travaux qui se limitent à l'étude des seuls récits qualifiés de «stratégiques »-récits dans lesquels se joue le sens du devenir de l'organisation - réduisent la complexité inhérente à l'exercice stratégique, au risque de le dénaturer, car ils méconnaissent le rôle des récits ordinaires dans la compréhension de la formation de la stratégie. Nous nous efforçons dans cet article, d'une part, de dépasser une vision univoque de la formation de la stratégie qui consisterait à la réduire à la conception d'un récit suffisamment pertinent pour être efficace, et d'autre part, d'élargir le champ d'analyse de la formation de la stratégie de façon à y inclure les récits ordinaires qui fondent la récursivité de la vie organisationnelle.

Nous montrons dans un premier temps que le mouvement d'importation de la thématique du récit dans les recherches en stratégie a généré un foisonnement de travaux particulièrement difficile à appréhender puisque, selon la perspective retenue, le champ sémantique couvert par les mêmes termes - conversations, récits, textes, discours - diffère largement, et qu'en outre, les relations entre ces différents types de productions organisationnelles sont conçues selon des modalités en apparence irréconciliables. Dans un second temps, en nous appuyant à la fois sur les acquis de l'approche narrative des organisations et les apports de la théorie du texte élaborée par Ricœur, nous intégrons à l'analyse de la formation de la stratégie les récits ordinaires, productions narratives destinées à faire sens de l'expérience quotidienne, qui portent et construisent la connaissance nécessaire au déroulement même des activités 
organisationnelles. La nature textuelle des productions stratégiques de l'organisation nous conduit à approfondir le rôle fondamental de la lecture, entendue comme processus de compréhension et de consommation des textes, dans la formation de la stratégie. Sur ces bases, nous proposons dans un troisième temps, une modélisation bi-modale de la formation de la stratégie. Nous détaillons d'une part, comment les textes stratégiques se constituent récursivement à partir des récits ordinaires qui informent les pratiques quotidiennes et, d'autre part, nous éclairons les relations qu'ils entretiennent avec l'ensemble des textes produits par les institutions et les parties prenantes concernées par le devenir de l'organisation. Le texte stratégique est ainsi inscrit dans un double rapport, d'une part, avec le contexte - l'ensemble des textes précédents avec lesquels il est relation -, et d'autre part avec la situation l'ensemble des activités ordinaires de l'organisation dont il rend compte et qu'il contribue à organiser. La mise en regard des textes stratégiques, des contextes institutionnels et des situations organisationnelles, nous permet de rendre compte du rôle médiateur des textes stratégiques et de clarifier l'ensemble des modalités concourant à la formation des textes stratégiques.

I - La délicate importation du récit en stratégie : diversité des projets d'investigation

Le récit connaît actuellement un engouement certain en management stratégique. Toutefois, le foisonnement de travaux sur ce thème selon des approches conceptuelles et des traditions de recherche très différentes, rend la tâche de présentation du champ particulièrement ardue. Au risque de simplifier de façon excessive les subtilités de certains travaux, nous proposons de retenir trois perspectives principales :

\section{- Une visée fonctionnaliste : un bon méta-récit, un instrument au service de la stratégie}

Comme l'ont démontré de nombreux travaux d'analyse littéraire, la fonction même du récit consiste à organiser un ensemble d'événements et d'intervenants multiples dans une trame temporelle commune, acceptable et compréhensible (Adam, 1999). Ces travaux ont souligné la capacité du récit à relier de multiples points de vue interdépendants afin de produire une unité de sens. En réorganisant les événements vécus dans une trame temporelle, le récit préserve et construit la continuité de l'action : la perception des enjeux de la situation présente organise directement les événements passés qui sont remémorés et mis en scène au cours d'une nouvelle histoire. Le récit structure ainsi un ensemble de relations et crée de façon rétrospective du sens afin d'ordonner la perception du monde et de pouvoir agir sur lui. Cette faculté de générer du sens qui caractérise les récits a conduit les observateurs de l'organisation (chercheurs, intervenants, historiens) à s'intéresser aux histoires racontées en son sein. L'étude des organisations se fait à plus d'un titre à partir de ses histoires, ce que démontrent en particulier les travaux sur la culture et l'identité de l'entreprise qui se sont penchés très tôt sur les grandes (mythes, récits, discours) comme sur les petites histoires (story, storytelling, gossip, narratives) de l'organisation (Gabriel 2000).

Différents modèles formels sont convoqués pour définir les caractéristiques d'un bon récit, qui se doit d'être à la fois cohérent, pour gagner en crédibilité, et mobilisateur, pour faciliter sa réception et sa mise en œuvre ultérieure. En effet, la «narratologie » a démontré qu'au-delà de leur diversité apparente, les récits présentent des formes de base et des principes de 
composition communs. Il est alors tentant d'appliquer la narratologie aux productions stratégiques. Ainsi Christian (1999) puis Boudès (2004) mettent en lumière la proximité entre stratégie et récit, entre une bonne histoire, bien construite, qui ne sonne pas faux et une bonne stratégie. Mobilisant la notion de «schéma actantiel» de Greimas, ils identifient les caractéristiques structurelles d'un récit efficace. Cette perspective est sous-tendue par l'idée que les bons récits sont facteur d'efficacité dans la mise en œuvre des idées de leurs auteurs et conduit à prôner la conception de récits bien construits et cohérents pour accroître l'efficacité du management stratégique. En considérant que la construction d'un bon récit suppose une intentionnalité globale - une maîtrise totale de la conception de l'intrigue jusqu'à son dénouement, ces travaux estiment que le récit ne peut rendre compte de certains phénomènes organisationnels comme les improvisations ou les activités les plus routinières, auxquelles il semble difficile d'attribuer une forme d'intentionnalité, même très partielle. Pour les tenants de cette approche, les activités ordinaires se développent au fil des conversations, registre discursif aisément différenciable de celui du récit, qui lui, constitue une unité discursive cohérente et aboutie caractérisée par une situation initiale, un événement déclencheur, une intrigue et un dénouement....

Dans cette perspective, l'exercice stratégique constitue une production narrative d'un type bien particulier puisqu'il s'agit d'élaborer un grand récit ou méta-récit qui assure une fonction intégrative permettant aux dirigeants de réorganiser les événements passés en fonction d'un projet de développement plausible. Ce que l'on retient du récit est sa capacité à construire un message persuasif et mobilisateur pour faciliter la mémorisation et l'apprentissage ou parvenir à convaincre l'ensemble des parties prenantes de la pertinence d'une stratégie. Toutefois, la qualité intrinsèque du méta-récit aurait-elle le pouvoir de dissoudre les difficultés inhérentes à l'exercice stratégique? En outre, la question de l'efficacité d'un méta-récit stratégique intégrateur peut-elle être posée sans intégrer la part d'interprétation potentielle apportée par les récepteurs auxquels il s'adresse?

\section{- Une visée communicationnelle : enjeux liés à la dynamique texte / conversation}

Barry et Elmes (1997) ont les premiers ébauché une analyse critique de la stratégie en déconstruisant minutieusement les discours stratégiques élaborés dans les organisations, et en particulier ceux produits par les dirigeants. En effet, la stratégie n'est pas seulement imaginée, instrumentée : elle consiste en un ensemble d'histoires racontées par des personnes clés, le plus souvent ses dirigeants, à d'autres personnes, - les actionnaires, les membres de l'organisation, les parties prenantes... C'est pourquoi, ces auteurs soulignent avec raison la double nature de la stratégie - à la fois production narrative et processus de narration - par laquelle différentes histoires quant à la direction prise ou à prendre par l'entreprise (stories about directionality) sont reliées, mises à l'épreuve, renforcées ou affaiblies. Ils établissent la proximité entre le genre romanesque, celui des romans d'apprentissage et des fictions réalistes, et les processus de changement stratégique qui organisent les interactions entre différentes catégories d'acteurs. Avec une ironie certaine, ces auteurs vont même jusqu'à montrer le caractère épique, voire parfois hagiographique, de certains discours de dirigeants, des textes qualifiés de «stratégiques », mais aussi, de façon plus étonnante, des théories stratégiques.... 
Taylor et Robichaud (2004) considèrent que pour qu'une interaction puisse se développer entre les membres d'une organisation, il est nécessaire que ces derniers élaborent un texte, c'est-à-dire un langage partagé qui leur permette de poursuivre leur relation. Selon ces chercheurs, c'est bien la conversation, par sa capacité à produire une orientation mutuelle du comportement des acteurs en interaction, qui est première dans la production de l'organisation. Les textes constituent de ce fait des productions secondaires qui formalisent les interprétations formulées dans certaines situations types et contribuent à les pérenniser afin de rendre disponibles afin de cadrer les interactions futures. D'une part, les textes produits gardent la trace de l'organisation que les conversations ont permis de mettre en place et d'autre part, ils impriment leur marque sur l'action organisationnelle qu'ils contribuent à canaliser, directement ou indirectement en offrant des cadres interprétatifs permettant de faire sens des événements en cours. Ainsi, «a text may thus be spoken as well as written. " (Taylor \& Robichaud 2004, p. 401), ce qui rapproche directement l'usage que ces auteurs font de la notion de texte de celle de cadre d'interaction - inspirée de Erving Goffman - que nous avions mobilisée pour analyser le processus de formation de la stratégie (La Ville 2001 a).

Cette perspective interactionnelle souligne les enjeux en matière de communication propres à l'élaboration de la stratégie : en tant que production narrative, cette dernière se doit d'être convaincante, pertinente, comprise, acceptée par l'ensemble des parties prenantes... en vue de faciliter la phase de mise en œuvre. Giroux et Demers décrivent la dimension communicationnelle de ce processus conçu comme une "chaîne de conversations » disséminées dans le temps et dans l'espace et envisagent un mode dialogique de production du récit stratégique, "à travers une vision polyphonique de la communauté où plusieurs voix se font entendre, où l'hétéroglossia (la présence simultanée de plusieurs langues) est acceptée » (Giroux \& Demers, 1997, p.7). La formulation de la stratégie est alors conçue comme un processus narratif qui organise une polyphonie entre des logiques variées. La polyphonie ne reconnaît pas simplement la co-existence de différentes logiques mais surtout le fait que ces dernières s'informent et se définissent mutuellement. Dans cette perspective, l'importation de la notion de récit dans le champ du management dépasse la performance narrative du dirigeant ou du noyau stratégique pour inclure de façon plus ou moins délibérée dans la narration de multiples parties prenantes. Par la polyphonie plus ou moins développée qu'il organise, le processus de formulation de la stratégie n'est jamais maîtrisé par un seul auteur (Giroux 1998), ce qui rend l'exercice stratégique particulièrement difficile dans certains contextes organisationnels. Ainsi, la stratégie s'élabore progressivement par négociation dans le cadre d'une "méta-conversation », métaphore discursive qui est utilisée pour figurer l'ensemble du processus stratégique (Giroux 1998 ; Giroux \& Demers 1997). Sont alors distinguées la "méta-conversation de formulation », qui produit, à travers un phénomène de textualisation visant l'abstraction et la généralisation, une « représentation globale de l'organisation dans des discours définitoires », et « la méta-conversation de mise en œuvre » qui opérationnalise le texte dans des représentations locales et concrètes à travers une série de documents explicatifs de nature analytique ou l'application d'un nouvel ensemble de critères de performance.

Ces modèles ont le mérite de mettre en lumière la diversité des constructions langagières, discursives et textuelles qui concourent à la formation de la stratégie dans les organisations, et devraient conduire le chercheur en management stratégique a être particulièrement attentif aux modalités conversationnelles et textuelles spécifiques selon lesquelles s'organise l'exercice stratégique dans chaque organisation. Ainsi, le processus stratégique peut être 
compris comme un effort communicationnel permanent en vue de faire accepter de nouvelles orientations stratégiques à l'ensemble des parties prenantes.

\section{- Une visée critique : l'effet disciplinant des productions textuelles de la stratégie}

Avec un visée explicite plus critique - au sens de critical studies (Alvesson \& Deetz 2000) certains travaux (Czarniawska 1997 ; Putnam \& Cooren 2004) offrent un autre point de vue sur le rôle que jouent les récits dans la vie organisationnelle. Certains documents nécessaires au fonctionnement de l'organisation - tels les règlements, les procédures, les notes de service...-, peuvent être qualifiés de «textes ». Ces derniers ne sont alors pas définis comme le produit des interactions entre les membres de l'organisation, mais plutôt par leur caractère structuré et permanent, qui concourt à la pérennisation de l'organisation, puisque cette dernière se définit par les documents (records) qu'elle produit et qui en retour la constituent (McPhee 2004). En effet, certains discours sont sélectionnés, certaines paroles sont retranscrites, certaines histoires sont pérennisées et rejoignent l'ensemble des textes inscrits, conservés, mémorisés, qui constituent une caractéristique essentielle de l'organisation : sa dimension disciplinante.

Réintégrant explicitement ou implicitement parfois les analyses de Foucault (1971), ces travaux soulignent le pouvoir disciplinant des discours formels et des textes sur les pratiques ordinaires et les activités menées quotidiennement par les membres de l'organisation, par leur capacité à imposer et légitimer certains cadres interprétatifs aux dépens d'autres. Foucault prévient qu'il s'agit "de ne plus traiter les discours comme des ensembles de signes (d'éléments signifiants renvoyant à des contenus ou à des représentations) mais comme des pratiques qui forment systématiquement les objets dont ils parlent.» (Foucault 1969, p. 66 et 67) Les discours apparaissent alors comme des « objectivations subreptices » : en apparence, ils se construisent par induction et se bornent à désigner le monde ; en réalité, ils participent à la constitution des objets même, du monde lui-même. Les discours et les textes mobilisent des mécanismes de domination qui trouvent leur légitimité, par exemple dans la rationalité, la scientificité invoquée de la discipline. Ils se traduisent par des pratiques de surveillance et de contrôle mais visent aussi à influencer la subjectivité des managers, à constituer une part de leur identité. Hardy et Phillips (2004) mettent en lumière comment certains acteurs sont plus à mêmes que d'autres de mobiliser un capital économique, culturel et social pour légitimer leur intervention et le pouvoir qu'ils exercent sur les processus organisationnels. Dans cette perspective, la notion même de discours inclut de façon consubstantielle celle de domination et s'oppose aux récits ordinaires, fragmentaires, empreints de subjectivité et d'émotion, situés, qui se construisent au fil des interactions et des activités développées par les membres de l'organisation....

D'un point de vue pragmatique, Czarniawska (1997) s'intéresse aux activités quotidiennes des managers qui ont pour effet de produire en permanence l'organisation elle-même. Selon elle, deux types de conversations coexistent au sein des organisations : d'une part, celles qui permettent la confrontation d'expériences vécues ou d'actions entre interlocuteurs, et celles qui fabriquent des récits en-dehors du champ de l'expérience vécue par l'individu en lui imposant des normes de comportement, de décision, etc. Cette coexistence se traduit par une compétition acharnée entre un ordre « Fort» - celui du Discours - qui renforce sa domination 
institutionnelle en contrôlant les interprétations, et un ordre «faible » - celui des récits ordinaires - simplement désireux de faire sens des activités quotidiennes... Son travail dénote une ironie certaine en ce qui concerne l'impact réel de l'activité des stratèges sur la vie effective de l'organisation car la stratégie y est indirectement dépeinte comme une construction discursive relativement artificielle, éloignée des réalités vécues par les membres de l'organisation et qui constitue en général un instrument de domination au service d'institutions légitimées... Sans impact sur le devenir des grandes organisations publiques et sans prise sur les activités quotidiennes qui s'y déroulent, la stratégie s'apparente à une ritualisation souvent vide de sens... (La Ville \& Mounoud 2003) La sévérité de ce constat s'explique en partie par le fait qu'il concerne principalement des organisations publiques où la distance entre les discours officiels et les pratiques effectives est parfois ressentie comme incommensurable par les managers et les acteurs sur le terrain.

En effet, l'idée que la stratégie comporte une dimension disciplinante, souligne l'importance des enjeux de pouvoir et de légitimation qui se font jour dans l'exercice stratégique. Car le langage utilisé quotidiennement dans l'organisation est lui-même porteur de logiques de domination qui s'infiltrent pour orienter durablement les interactions ordinaires et les activités quotidiennes et légitimer les relations de pouvoir dans l'organisation (Hardy 2004).

L'importation de la notion de récit dans le champ du management stratégique a provoqué un foisonnement de perspectives particulièrement difficile à maîtriser puisque, selon la visée poursuivie, le champ sémantique couvert par les mêmes termes - conversations, récits, textes, discours - diffère largement, et qu'en outre, les relations entre ces différents types de productions organisationnelles sont conçues selon des modalités parfois totalement irréconciliables. Toutefois, cette lecture de la façon dont la notion de récit a été importée en stratégie, parfois sur un plan métaphorique, parfois avec une volonté empirique forte, permet d'appréhender à quel point la formation de la stratégie met en jeu des modalités complexes d'interaction entre des productions organisationnelles de nature très variée qu'il convient de cerner avec précision.

\section{II - Les textes stratégiques au cour d'une dialectique d'écriture et de lecture}

En suivant Czarniawska, nous considérons que la vie quotidienne au sein de l'organisation prend corps à travers un ensemble de récits ordinaires grâce auxquels les individus sélectionnent des événements, organisent des temporalités, typifient des personnages clés, construisent des rapports d'identité, structurent leur propre expérience, élaborent et transforment leurs interprétations. Considérant à la suite de Bruner, "qu'il existe une disponibilité, ou une prédisposition à organiser le vécu sous forme narrative en construisant des intrigues » (Bruner 1991, p. 59), nous estimons que la continuité de l'existence ne peut s'appréhender autrement qu'à travers le récit de cette même existence, processus narratif grâce auquel le locuteur façonne sa propre identité et en octroie une à ceux qu'il inclut ou exclut de son récit. Car c'est bien par la parole énoncée, échangée et retenue entre ses membres que se construit et se pérennise l'organisation : le langage, la parole et la mise en récit, la production de simples histoires, de narrations ordinaires au fil des événements vécus, sont consubstantiels au processus même d'organisation. La construction des activités, des connaissances et des identités au sein de l'organisation est ainsi structurée au fil d'un entrelacs complexe de 
processus narratifs qui est toujours spontané, lié à des événements imprévus, et aussi en partie socialement organisé (Czarniawska 1998).

Le devenir de l'organisation se construit au fil d'une narration collective, dispersée et fragmentée, faisant l'objet d'élaborations partielles, de transformations plus ou moins profondes et d'inscription plus ou moins pérennes dans des textes. Or, la stratégie, envisagée comme volonté d'orienter le développement futur de l'organisation et de contrôler les activités des membres dans l'organisation pour les conformer à ces orientations, se traduit en partie par la production de textes. Toutefois, notons bien dès à présent que tous les textes produits dans et par l'organisation ne peuvent être qualifiés de "stratégiques » et, ensuite que le processus stratégique lui-même ne se limite pas aux modalités de fabrication des textes dits « stratégiques », ni au simple suivi de l'effet qu'ils produisent dans l'organisation.

\section{1 - La production de textes stratégiques appelle la lecture...}

Pour analyser la dynamique des textes dans l'organisation, nous prenons appui sur le travail philosophique de Paul Ricœur pour qui la notion de texte recouvre une catégorie limitée de signes : ceux qui comportent une forme de fixation comparable à celle qu'opère l'écriture (Ricœur 1986, p. 205) car cette dernière permet la conservation et la linéarisation de la conversation, en l'autorisant à s'affranchir des limites de la parole en situation. S'il est vrai que toute écriture se rajoute à quelque parole antérieure, elle introduit toutefois un changement radical dans le rapport aux énoncés. : la parole renvoie à ce que Ricœur appelle la " référence ostensive », c'est-à-dire l'objet à propos duquel on s'exprime et que l'on peut presque montrer. L'écriture, quant à elle, crée un rapport différent de celui d'interlocution : le lecteur est absent à l'écriture, l'auteur à la lecture. Le texte procède donc d'une occultation simultanée du lecteur et de l'auteur. Dans cette acception, la nature particulière du texte est soulignée : par sa fixation sur un support, par la dissociation entre l'intention de l'auteur et l'intention du texte, par le déploiement de références «non ostensives » et par le fait qu'il n'a plus d'auditeur visible mais un ensemble de lecteurs inconnus, le texte doit être différencié du discours en situation de co-présence, du domaine de la conversation ordinaire.

En conséquence, "le texte attend et appelle la lecture" (Ricœur 1986, p.170). Ricoeur souligne qu'il y a deux façons d'envisager la lecture : "Expliquer c'est dégager la structure c'est-à-dire les relations internes de dépendance qui constituent la statique du texte. Interpréter c'est prendre le chemin de pensée ouvert par le texte, se mettre en marche vers l'orient du texte " (Ricœur, $1986: 175)$ et propose de dépasser ce dualisme pour mettre à jour la dialectique qui caractérise l'activité même de lecture. En effet, la véritable destination de la lecture c'est d'effectuer un mouvement dialectique entre ces deux attitudes pour produire une interprétation. C'est le caractère ouvert du texte qui est alors souligné : des références sont offertes par le texte qui proposent un "monde » et construisent un nouveau projet et ce qui rend la lecture possible c'est que le texte n'est pas fermé sur lui-même mais ouvert sur autre chose. La lecture apparaît alors comme une activité créatrice, qui prolonge la création propre de l'écriture : "Lire, c'est en tout hypothèse enchaîner un discours nouveau au discours du texte » (Ricoeur 1986, p. 220). 
Cette analyse de la créativité de la lecture est également partagée par Certeau qui justifie l'intérêt de mieux analyser l'activité de lecture par l'importance considérable qu'a pris l'écriture dans la société contemporaine, "de plus en plus écrite, organisée par le pouvoir de modifier les choses et de réformer les structures par des modèles scripturaires (scientifiques, économiques, politiques), muée peu à peu en "textes " combinés (administratifs, urbains, industriels, etc.) (Certeau 1990, p. 243). Son analyse tend à montrer combien la lecture n'est pas une activité passive alors qu'elle semble pourtant "le point maximal de la passivité » et souligne combien la lecture modifie son objet, réinvente autre chose que l'intention du texte et construit un "monde » différent, celui du lecteur qui s'insinue dans la place de l'auteur pour " rendre le texte habitable » (Certeau 1990, p. XLIX). Lors des instants passés à lire, le lecteur effectue " une réappropriation du texte de l'autre : il y braconne, il y est transporté, il s'y fait pluriel » (Certeau 1990, p. XLIX). Appliquant ce point de vue aux rapports entre consommation et production, Certeau montre que, si d'un côté, la consommation suppose l'acceptation d'une offre imposée de produits, en revanche d'un autre côté, les consommateurs ne sont jamais passifs ou dociles, ils prennent plaisir à consommer, se considèrent bien souvent libres et créatifs dans leurs pratiques. Sous l'apparente banalité de gestes ordinaires et d'actions fortement routinisées, se cache une extraordinaire créativité souvent ignorée par la théorie. Les individus font preuve d'une grande capacité de bricolage, d'inventivité en termes d'astuces et de stratagèmes, pour élaborer leur façon singulière d'accomplir les activités quotidiennes : cuisiner, déambuler dans une ville, faire ses achats, etc. Analysés superficiellement, certains comportements routiniers semblent révéler une forme de soumission alors même qu'ils sont le lieu d'expérimentation de formes de résistance et de créativité. Les rapports entre lecture et écriture sont de même nature : les textes, tels des biens mis sur le marché, sont produits par des fabricants qui les imposent aux consommateurs, les lecteurs, ce qui oriente de façon déterminante - mais jamais définitive - leur signification et leur usage potentiel.

En transposant cette métaphore, la formation de la stratégie peut être comprise comme un processus créatif permanent incluant non seulement ce que les stratèges produisent - ou écrivent - (des textes, des budgets, des plans, des matrices, des stratégies...) mais également la façon dont les membres de l'organisation consomment - lisent - ces productions. Ainsi, il convient de s'intéresser à la façon dont les différentes acteurs au sein de l'organisation lisent, utilisent et transforment les textes stratégiques dans le cours de leurs activités quotidiennes.

\subsection{La lecture : l'apport créatif du registre tactique à la formation de la stratégie}

L'analyse de la lecture proposée par Certeau s'ancre dans la distinction qu'il opère entre "stratégie » et "tactique ». "J'appelle "stratégie 》 le calcul des rapports de forces qui devient possible à partir du moment où un sujet de vouloir et de pouvoir est isolable d'un " environnement ». Elle postule un lieu susceptible d'être circonscrit comme un propre et donc de servir de base à une gestion de ses relations avec une extériorité distincte. La rationalité politique, économique ou scientifique s'est construite sur ce modèle stratégique. »" (Certeau 1990, p. xlvi) Les stratégies cachent sous une mise en scène rationnelle et des calculs objectifs, leur rapport direct avec le pouvoir qui les soutient et les institutions qui les légitiment. La stratégie est intimement liée à la notion de pouvoir et révèle sa visée disciplinante car elle s'impose par une mise en scène rationnelle. "J'appelle au contraire "tactique " un calcul qui ne peut pas compter sur un propre, ni donc sur une frontière qui 
distingue l'autre comme une totalité visible. La tactique n'a pour lieu que celui de l'autre. Elle s'y insinue, fragmentairement, sans le saisir en son entier, sans pouvoir le tenir à distance. Elle ne dispose pas de base où capitaliser ses avantages, préparer ses expansions et assurer une indépendance par rapport aux circonstances. » (Certeau 1990, p. xlvi) Dans cette définition, la tactique ne se résume pas à l'intendance, toujours censée suivre la stratégie. La tactique est par nature l'expression d'une créativité singulière dans un ensemble de micro décisions, d'actes de résistance, peu coordonnés, mais destinés à échapper au pouvoir disciplinant de la stratégie.

Ces définitions, en revenant aux sources mêmes de l'art militaire, redonnent toute sa place à la tactique, notion que la communauté de gestion - les chercheurs tout comme les praticiens semble avoir trop souvent réduite à un simple enjeu de mise en œuvre de la stratégie édictée. Les pratiques d'espionnage et de contre-espionnage, les diverses formes de guérilla sporadiques, susceptibles de créer de l'instabilité et de parvenir à renverser à terme les rapports de force, sont des figures liées à l'art militaire qui ont été largement ignorées par les modélisations de gestion, sauf peut-être dans le champ de l'entrepreneuriat où le héros néoschumpétérien se bat contre les institutions et les instances de pouvoir pour tenter de faire vivre son innovation, en s'immisçant subrepticement dans des espaces dominés par des acteurs puissants, en s'appuyant sur des réseaux relationnels relativement secondaires, en choisissant des voies marginalisées pour expérimenter ses idées et donner progressivement corps à son projet (La Ville 2001 b).

Or, la tactique est bien l'expression d'une créativité, d'une capacité à «faire avec », à subvertir des règles imposées afin de se créer des marges de manœuvre, si minimes soientelles. La tactique comporte de façon irréductible une part de créativité à peine consciente, qui se donne à voir sous la forme de bricolages multiples, d'inventions particulièrement ingénieuses... Cette créativité est perceptible dans les tentatives d'associer les actions et les événements ou dans les négociations quant à leur signification entre les membres de l'organisation, c'est-à-dire, finalement, dans la dynamique des récits ordinaires élaborés au fil de la vie quotidienne de l'organisation. Le registre narratif est bien celui qui permet à l'art tactique de s'exprimer, de s'essayer à de nouvelles possibilités, d'improviser, et de résister à la domination d'une totalité disciplinante martelée par les textes stratégiques. Certeau identifie trois lieux où se donne à voir cette créativité tactique des récits ordinaires: les « jeux » spécifiques à chaque société qui formalisent les règles organisatrices des " coups »; les « récits de partie » qui enseignent les tactiques disponibles; les contes et légendes qui exposent les bons et mauvais tours utilisables chaque jour. Ainsi, cette part de consommation que comprend l'activité stratégique, du fait de sa dispersion, de ses actions subreptices, des astuces et de la ruse qu'elle comporte, est souvent hors d'atteinte pour le chercheur - et le manager - et c'est pourquoi elle semble trop souvent occultée dans la plupart des modélisations.

Cette lecture - ou consommation - des textes stratégiques constitue elle-même une production de deuxième ordre, ce que Certeau appelle une fabrication, c'est-à-dire un discours qui se surajoute à l'intention des textes stratégiques. En effet, les employés n'adhèrent pas, n'épousent pas ou ne partagent pas la vision stratégique ou les mondes produits par les stratèges ou la direction générale. Dans leurs activités quotidiennes, les managers et les employés apprennent, expérimentent, font preuve d'esprit critique pour prendre des micro- 
décisions, qu'elles soient destinées à mettre en œuvre les orientations stratégiques, ou bien à résister en pratique aux changements imposés par de nouveaux projets stratégiques. La conceptualisation proposée par Certeau permet ainsi d'appréhender la complexité et la créativité de la formation de la stratégie et de repenser le lien inextricable entre "l'émergent » et «le délibéré »: d'une part, des processus d'institutionnalisation accompagnent et soutiennent l'émergence de discours organisationnels intégrateurs, les textes stratégiques, et d'autre part, d'innombrables lectures qui se développent de façon désordonnée et incontrôlée, pour donner progressivement corps à une multiplicité de tactiques qui constituent le registre de la pratique stratégique...

\subsection{Le texte stratégique dans une dynamique de médiation entre contexte et situation}

L'ensemble de ces réflexions nous conduit à considérer que les textes stratégiques constituent des formes médiatrices grâce auxquelles les acteurs de l'entreprise sont capables d'exercer une forme de réflexivité pour comprendre leurs situations, attribuer du sens à leurs actions et envisager le devenir de leur organisation dans son contexte. Les textes stratégiques ainsi conçus s'inscrivent dans un double rapport, d'une part avec le contexte - l'ensemble des textes précédents avec lesquels il entre en relation, - et d'autre part, avec la situation l'ensemble des activités et pratiques ordinaires de l'organisation dont il rend compte et qu'il contribue à organiser.

\subsection{1 - Des médiateurs entre la production et la consommation de la stratégie}

La production ou l'écriture d'un texte stratégique repose clairement sur un ensemble de prérequis scripturaires : pour être qualifié de «stratégique », le texte doit convoquer un certain nombre de normes, de règles, de critères qui le rapportent explicitement à la discipline du management et à différents genres admis dans les cercles stratégiques : rapport d'activité, business plan, plan de développement, plan de recentrage ou de redéploiement d'actifs, etc. Des processus d'institutionnalisation accompagnent et soutiennent la production des textes stratégiques tout en permettant de les différencier des autres productions textuelles de l'organisation. Les MBA, les cursus académiques spécialisés, les cabinets de conseil et les associations professionnelles contribuent à légitimer la communauté des stratèges et à accroître leur capacité de domination dans la société contemporaine... L'activité stratégique de l'organisation se donne à voir à travers un ensemble de productions textuelles propres qui ont pour but de montrer la pertinence du projet stratégique et sa maîtrise par le noyau stratégique, tout en facilitant sa communication auprès des parties prenantes impliquées dans le devenir de l'organisation.

Mais, au-delà de cette dynamique d'écriture qui préside à leur production, les textes stratégiques se caractérisent par leur plurivocité : leur signification est comme en suspens et chaque texte, du fait de la distanciation provoquée par sa forme écrite, ouvre sur une pluralité de lectures et de constructions en fonction des situations dans lesquelles se trouvent ses différents lecteurs. A travers leur travail d'interprétation des textes stratégiques, ce que les acteurs de l'organisation s'approprient, c'est une proposition de relation au monde. Ce processus d'appropriation, qui se déploie sur le registre narratif est complexe, touffu, foisonnant, et fait l'objet de maintes remises en causes, de négociations, d'échanges de 
vues.... Les récits ordinaires donnent à voir comment s'opère la lecture, à travers la mobilisation d'innombrables tactiques, fragmentaires, instantanées, opportunistes, improvisées et qui se développent de façon désordonnée ou incontrôlée.... "Ces tactiques manifestent aussi à quel point l'intelligence est indissociable des combats et des plaisirs quotidiens qu'elle articule. (...) du fait de son non-lieu, la tactique dépend du temps, vigilante à y « saisir au vol » des possibilités de profit. Ce qu'elle gagne, elle ne le garde pas. Il lui faut constamment jouer avec les événements pour en faire des "occasions ».» (Certeau 1990, p. XLVII). Par nature incapables de capitaliser volontairement leurs acquis, de maitriser la dimension temporelle et le cours de événements, ces tactiques propres à l'art de la lecture sont néanmoins susceptibles de produire des effets stratégiques tangibles et parfois irréversibles, tels que des délais de mise en œuvre, des détournements d'objectifs, des diversions opérationnelles, des rejets symboliques plus ou moins perlés, de subversion des règles ou des principes d'autorité...

Figure 1 : Les textes stratégiques : médiateurs à la croisée d'une double dualité

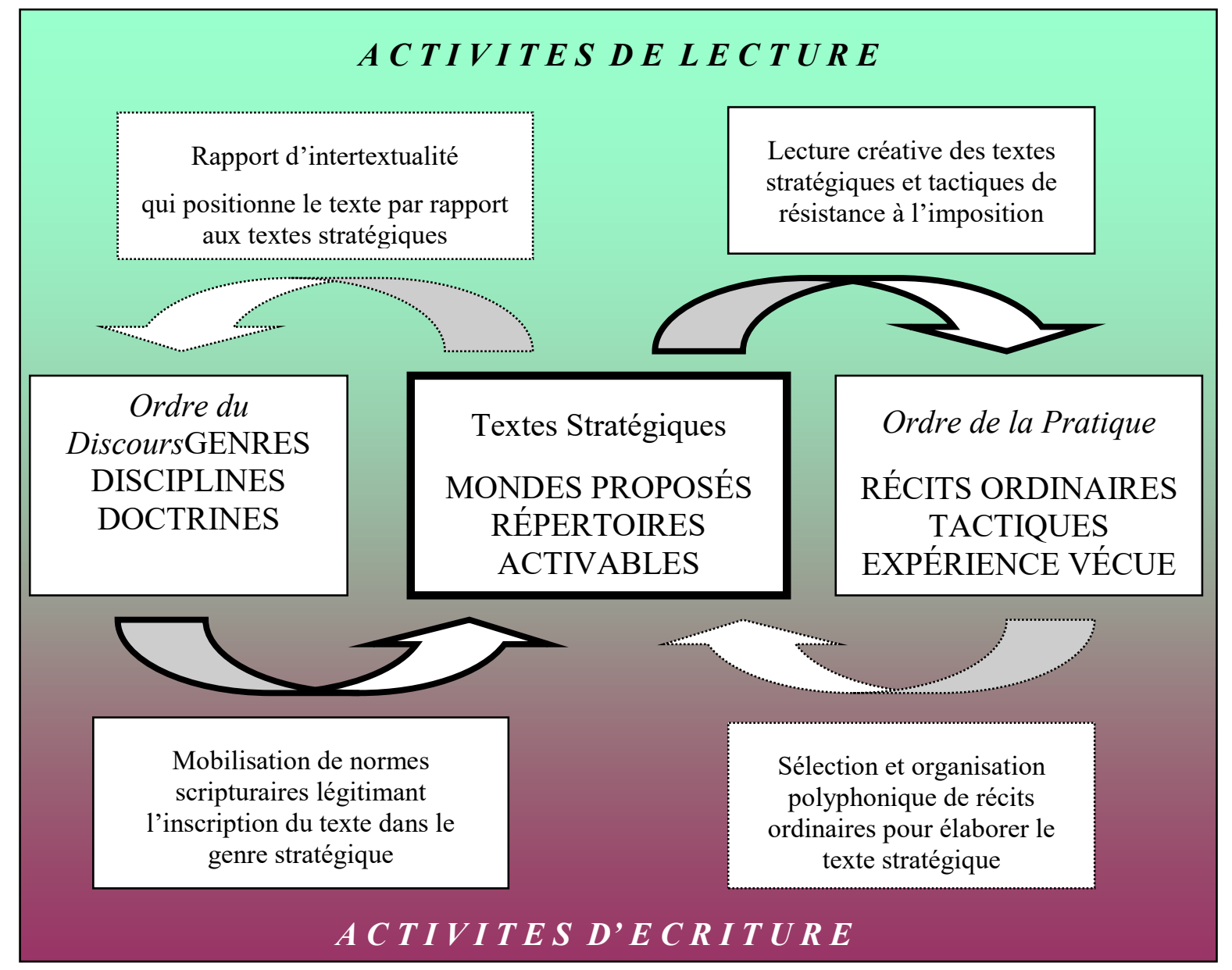




\subsection{2 - Des médiateurs qui organisent les rapports d'intertextualité et de polyphonie}

D'autre part, les textes stratégiques sont enchâssés dans des relations dites de textualité, avec les textes stratégiques pré-existants, eux-mêmes régis par les genres institués de productions organisationnelles, par l'ordre du Discours qui s'appuie non seulement sur les avancées scientifiques de la discipline du management au sens large (Hardy, 2004), mais également sur un contexte élargi incluant l'existence d'objectifs et de stratégies qui ne sont pas purement économiques, mais reposent davantage sur les valeurs constitutives du système social local. L'activité stratégique étant directement enchâssée dans un environnement institutionnel, on ne peut ignorer qu'elle comporte inévitablement une dimension idéologique, car elle reproduit les inégalités de la société capitaliste, contribue à étendre les structures managériales occidentales et présente les objectifs des élites dominantes comme des buts universels. Du fait de leur inscription institutionnelle, les textes stratégiques proposent un monde qui comporte une dimension disciplinante fondée à la fois sur des normes scripturaires légitimées et des systèmes de valeurs institutionnalisés.

D'une part, parce qu'ils mettent en scène une proposition de relation au monde, les textes stratégiques ne peuvent ignorer la multiplicité de voix, d'actions et de narrations qui se font écho les unes aux autres au sein de l'organisation (Czarniawska ce numéro; Giroux \& Demers 1997). La production même des textes stratégiques organise ainsi un rapport polyphonique entre des voix qui demeurent irréductiblement singulières et se développent de façon autonome, tout en se répondant, en s'opposant, en se renforçant pour contribuer à une nouvelle proposition de monde (O’Connor 2000 ; Hardy 2004). La formation de la stratégie procède d'une mise en pratique des apprentissages stratégiques qui ne relève pas uniquement de l'ordre du discours ni de celui de la conversation, mais intègre également un ensemble de savoirs incorporés dans le cadre des activités et tactiques plus ou moins tacites quotidiennement pratiquées par les membres de l'entreprise. Mais au-delà, les pratiques ordinaires des managers et les récits qui les sous-tendent, par la résistance qu'ils expriment, nourrissent en permanence l'inventivité des actions organisationnelles et composent une part irréductiblement créative de l'activité stratégique en perpétuelle émergence... Soit ces pratiques sont progressivement reconnues, dénommées, et définies pour être intégrées au texte stratégique, soit elles ne sont pas visibles, - car peut-être menées en perruque ou en-dehors des voies officielles - ou demeurent mal appréhendées et restent en-dehors des textes stratégiques produits par l'entreprise. C'est dans le registre polyphonique que le texte stratégique organise, que s'articulent les éléments constitutifs du registre tactique et qu'est reconnu son apport créatif à la formation du projet stratégique.

\section{Conclusion : Vers un modèle linguistique de l'action stratégique?}

Notre réflexion, soucieuse de montrer que la formation de la stratégie met en jeu des modalités complexes d'interaction entre des productions organisationnelles de nature contrastée, s'ouvre sur un modèle de l'action stratégique prenant le texte comme modèle de l'action «sensée ». Cette dernière " devient objet de science seulement sous la condition d'une sorte d'objectivation équivalente à la fixation du discours par l'écriture.» (Ricœur 1986, p. 213). Ricœur rapproche ainsi l'action ordinaire de la parole et de la conversation : l'action est elle aussi une manifestation ancrée dans le présent, proche en cela de la structure 
de l'acte de langage et de l'énonciation. A l'inverse, l'action sensée, à laquelle nous rattachons l'action stratégique, se pérennise, laisse une trace et devient un élément marquant, susceptible d'être remémoré. Une action imprime sa marque quand elle contribue à l'émergence de configurations signifiantes, elle relève d'un processus d'enregistrement qui la transforme en «document» ou en «archive» de l'action organisationnelle, ce qui la rapproche du texte et la distingue de la conversation. L'action sensée procède d'une émancipation à l'égard du contexte situationnel initial et développe des significations qui peuvent être actualisées ou remplies dans des conditions autres, dans d'autres contextes organisationnels. En conséquence, l'analyse de la formation de la stratégie ne doit pas se centrer uniquement sur les récits, les conversations ou les interactions qualifiées de " stratégiques », mais plutôt chercher à cerner les modalités d'interaction entre les modes narratifs et discursifs qui caractérisent la formation de la stratégie. L'ordre descendant du discours et l'ordre ascendant de la narration, ces deux modalités de l'agir stratégique, font du texte stratégique leur point de rencontre, d'intersection, d'articulation et permettent de rende compte de la complexité de la formation de la stratégie. Ainsi, le contexte domine et informe le texte stratégique car il lui fournit des règles et des repères d'écriture pour se constituer; en retour, le texte stratégique, nourri pour partie des récits ordinaires sur les pratiques effectives des membres de l'organisation, tout à la fois s'impose et s'offre par la lecture à la consommation créative et résistante des narrations ordinaires. L'action humaine est ouverte à quiconque sait lire. » (Ricœur, 1986: 220) C'est à notre avis dans ce double rôle de médiation que le texte stratégique prend son sens et montre son efficace.

\section{Références}

M. Alvesson and S. Deetz, Doing Critical Research Management, Sage, London, 2000

J.M. Adam, Le récit, Que Sais-Je, $6^{\circ}$ édition, Presses Universitaires de France, Paris, 1999

T. Boudes, «La formulation de la stratégie comme mise en récit », Management International, Vol. 8 , No. 2, Hiver, p. 25-31, 2004

J. Bruner, Car la culture donne forme à l'esprit - De la révolution cognitive à la psychologie culturelle, Traduit de l'Anglais par Yves Bonin, Editions Eshel, Paris, 1991

D. Christian, A la recherche du sens dans l'entreprise... Compter, raconter ? La stratégie du récit, Maxima, Paris, 1999

B. Czarniawska, A narrative approach to organization studies, Qualitative Research Methods Series, No. 43, Sage Publications, Thousand Oaks, 1998

B. Czarniawska, Narrating the Organization - Dramas of Institutional Identity, The University of Chicago Press, Chicago, 1997

M. De Certeau, L'invention du quotidien - Arts de faire, Editions Gallimard, Paris, 1990

V.I. De La Ville, «L'actualisation collective des pratiques stratégiques », chapitre 4, in Drisse (Ed.), Le management stratégique en représentations, Ellipses, Paris, p. 113-148, 2001 a

V.I. De La Ville, «L'émergence du projet entrepreneurial: apprentissages, improvisations et irréversibilités ", Revue de l'Entrepreneuriat, Vol. 1, No. 1, p. 43-59, 2001 b

V.I. De La Ville et E. Mounoud, «Between Discourse and Narration: How can Strategy be a Practice ? », in Czarniawska B. \& Gagliardi P. (Eds.), Narratives We Organize By, John Benjamins, Amsterdam, p. 95-113, 2003

M. Foucault, L'archéologie du savoir, Gallimard, Paris, 1969 
M. Foucault, L'ordre du discours, Nrf, Gallimard, Paris, 1971

Y. Gabriel, Storytelling in organizations, Facts, Fictions and Fantasies, Oxford University Press, New York, 2000

N. Giroux, «La communication dans la mise en œuvre du changement », Management International, Vol. 3, No. 1, p. 1-14, 1998

N. Giroux et C. Demers, "Communication organisationnelle et stratégie», Management International, Vol. 2, No. 2, p. 17-32, 1997

C. Hardy, « Scaling up and bearing down in discourse analysis: questions regarding textual agencies and their context », Organization, Vol. 11, No. 3, p.415-425, 2004

R. McPhee, «Text, Agency and Organization in the Light of Structuration Theory », Organization, Vol. 11, No. 3, p. 355-371, 2004

E. O'Connor, «Plotting the organization: The embedded narrative as a construct for studying change», Journal of Applied Behavioral Sciences, No. 36, p. 174-192, 2000

Y. Pesqueux, «Des histoires à l'histoire dans la formation de la stratégie : l'homme est un raconteur d'histoires », in Drisse (Ed.), Le management stratégique en représentations, Ellipses, Paris, 2001

L.L. Putnam and F. Cooren, «Alternative perspectives on the role of text and agency in constituting organizations », Organization, Vol. 11, No. 3, p. 323-333, 2004

P. Ricoeur, Du texte à l'action - Essais d'herméneutique II, Editions du Seuil, Paris, 1986

J.R. Taylor and D. Robichaud, "Finding the organization in the communication: discourse as action and sensemaking », Organization, Vol. 11, No. 3, p. 395-413, 2004 